\title{
Incertitudes sur les débits de crue
}

\author{
Uncertainty of flood discharge estimates
}

\author{
Michel Lang(1), Christian Perret ${ }^{(2)}$, Elodie Renouf ${ }^{(1)}$, Eric Sauquet(1), André Paquier ${ }^{(1)}$ \\ ${ }^{(1)}$ CEMAGREF Lyon, 3 bis quai Chauveau, 69009 Lyon \\ Tél : + 33 (0)4 722087 64, Fax : + 33 (0)4 784778 75, e-mail : michel.lang@lyon.cemagref.fr \\ (2) EDF DTG, 21 avenue de l'Europe, 38040 Grenoble Cedex 9, e-mail : christian.perret@edf.fr
}

Uncertainty on flood discharge estimates are linked to errors on flood stage, discharge measurement and rating curve extrapolation. According to a set of several hundred of French hydrometric stations, the standard uncertainties are of the order of $1 \%(i . e \pm 5 \mathrm{~cm})$ on flood water level, and between 3 to $5 \%$ on discharge measurement, with larger values when flow velocity or gradient of water raising are important. More than half of the rating curves have not been gauged more than a 2 year flood. Errors on non gauged discharges can be very large when the hydraulic properties of flow are not taken into account (non linear relationship between stage and discharge, discontinuity of flow between main channel and flood plain). The final uncertainty on discharge estimate based on hydraulic extrapolation of the rating curve is about $\pm 15 \%$ for the 10 year flood, except for complex hydraulic situations or without any gauging values.

\section{MOTS CLEFS}

Incertitudes, Débit, Crue, Jaugeages, Courbe de tarage, Modélisation hydraulique

\section{I 口 INTRODUCTION}

La chaîne de traitement permettant la production de séries hydrométriques repose sur l'établissement de courbes de tarage reliant le débit à la hauteur d'écoulement. La construction et le contrôle de ces courbes sont basés sur l'exploitation de jaugeages (mesure hauteur-débit) où le débit est mesuré directement en explorant le champ de vitesses ou indirectement par dilution d'un traceur chimique. Or les données de jaugeage en période de crue sont peu abondantes du fait de la difficulté d'être sur le terrain au bon moment (information sur l'existence d'une crue, délai minimum pour arriver sur le site, contraintes d'organisation la nuit et le week-end), ou de l'impossibilité d'effectuer la mesure (coupure des voies d'accès à la rivière, vitesses trop élevées pour le matériel, danger important pour les opérateurs...).

On constate des erreurs méthodologiques dans l'extrapolation de la courbe de tarage en période de crue, du fait de la non prise en compte des propriétés hydrauliques des écoulements (extrapolation linéaire de la courbe de tarage au lieu d'une relation puissance $Q=a H^{b}, b \neq 1$; relation non univoque due à une influence aval ...) ou de discontinuités dans les écoulements (débordement lit mineur-majeur, contournement de la station par des bras de décharge, mise en charge d'ouvrages ...). Par ailleurs se posent plusieurs questions sur la mesure en période de crue de la hauteur au droit des échel- les hydrométriques. Les fortes vitesses d'écoulement peuvent induire des phases de transport solide avec une modification du niveau du fond du lit, des pertes de charge locales au droit des capteurs peuvent fausser la représentativité de la mesure, et le capteur peut avoir été endommagé ou emporté au-delà d'un certain seuil de niveau. Enfin la modification des conditions d'écoulement à l'échelle de plusieurs années (envasement progressif, incision du lit, travaux hydrauliques ...) induit la définition de plusieurs courbes de tarage, dont il faut s'assurer de la pertinence en crue (bon raccordement) et du bon choix des périodes de validité.

L'ensemble de ces points induit une qualité très variable des données hydrométriques, suivant la configuration hydraulique du site, de la disponibilité de mesures de contrôle (jaugeages, topographie, laisses de crues), la gamme de débit et le niveau d'expertise dans l'établissement des courbes de tarage. Après un état des lieux sur le niveau d'extrapolation des courbes de tarage en France (section 2), nous présentons les niveaux d'incertitude rencontrés aujourd'hui pour la mesure des hauteurs (section 3) et la mesure des débits (section 4) des rivières en crue. Un rappel est fait sur les moyens de contrôle qui peuvent être à disposition tels que les ouvrages hydrauliques ou les modèles hydrologiques (section 5). Un travail spécifique d'expertise hydraulique d'une dizaine de courbes de tarage donne une indication de l'erreur d'estimation sur les débits de crue, au-delà des plus fortes valeurs jaugées (section 6). 


\section{E ÉTAT DE L'ART SUR L'EXTRAPOLATION DES COURBES DE TARAGE EN FRANCE}

Les courbes de tarage sont généralement établies à partir d'une série de jaugeages et présentent deux parties, la première sur le domaine des observations, plus ou moins précise selon la dispersion des jaugeages autour d'une courbe moyenne, la seconde extrapolée au-delà du plus fort jaugeage réalisé. Afin de pouvoir facilement comparer d'une station à une autre la limite de ce second domaine, nous avons calculé la période de retour $T$ de la valeur maximale de crue jaugée. Les données sur le plus fort jaugeage réalisé ont été extraites par le Schapi en 2005, sur un jeu de 443 stations hydrométriques. Elles ont été traitées avec la procédure CRUCAL de la banque HYDRO (ajustement de Gumbel effectué sur l'échantillon des valeurs de débit instantané maximal annuel) pour les stations disposant d'un nombre suffisant d'années d'observation pour estimer des quantiles de crue. Lorsque le débit maximal jaugé était inférieur au paramètre de position de la loi de Gumbel (période de retour $T<1$ an), nous avons comparé cette valeur au module interannuel QA, qui correspond approximativement au débit dépassé $30 \%$ du temps. Les autres valeurs obtenues ont été réparties dans quatre classes de période de retour: crue fréquente $(T<2$ ans), crue moyenne $(2 \leq T<10$ ans), crue forte ( $10 \leq T<100$ ans), crue rare ( $T \geq 100$ ans). (cf. échelle nationale d'intensité de crue, Lang et Claudet [2005]).

A l'issue de ce travail [Renouf et al., 2005], on dispose d'informations sur 325 stations hydrométriques, réparties majoritairement sur la zone Rhône Méditerranée Corse (RMC). Le tableau 1 et la figure 1 montrent que pour la majorité des stations, le débit correspondant à la hauteur maximale jaugée est de période de retour inférieure à 2 ans. Très peu de stations sont jaugées au delà de la décennale $(9 \%)$ et parmi ces stations, les grands bassins sont les mieux renseignés (crues plus lentes donc déplacements plus faciles à anticiper et à mesurer que les crues éclairs des petits cours d'eau). Les conclusions peuvent être biaisées par le jeu de stations utilisées (localisées pour l'essentiel dans le bassin $\mathrm{RMC}$ ) ou la fréquence de mise à jour de la base de données. Il reste à confirmer ces chiffres par une analyse étendue à l'ensemble du territoire français. D'autres traitements pourraient alors être envisagés (typologie par régime hydrologique, temps de réaction des bassins).

Tableau 1. Histogramme des classes de débit maximal jaugé.

\begin{tabular}{ccccccc}
\hline Classe & \multicolumn{2}{c}{$\mathbf{0}$} & $\mathbf{1}$ & $\mathbf{2}$ & $\mathbf{3}$ & $\mathbf{4}$ \\
\hline & $Q<Q A$ & $Q>Q A$ & {$[1 ; 2[$} & {$[2 ; 10[$} & {$[10 ; 100[$} & $T>100$ ans \\
\hline Effectif & 13 & 160 & 24 & 99 & 27 & 2 \\
\hline (\%) & 4,0 & 49,2 & 7,4 & 30,5 & 8,3 & 0,6 \\
\hline
\end{tabular}

\section{III — INCERTITUDES SUR LA MESURE DES NIVEAUX D'ÉCOULEMENT}

On rappelle que toutes les mesures de niveau sont rattachées à un repère fixe a priori immuable : l'échelle limnimétrique.

\section{III.1 La précision intrinsèque des capteurs}

Une étude réalisée à EDF DTG [Perret, 1993] a montré que les capteurs de niveau utilisés alors pouvaient en général être considérés comme performants en terme de précision. L'étude avait porté sur des capteurs piézorésistifs analogiques et numé- riques, bulle à bulle, ultra-sons dans l'air et dans l'eau, et s'était attachée à tester leur sensibilité aux facteurs d'influence suivants : température de l'eau, température de l'air, compatibilité électromagnétique et dans certains cas, conductivité. Les résultats ont montré que les capteurs piezorésistifs analogiques ou numériques étaient bien corrigés de l'influence de la température de l'eau (gamme $0{ }^{\circ} \mathrm{C} 25^{\circ} \mathrm{C}$ ). Le même constat a été fait pour le bulle à bulle, sensible quant à lui à la température de l'air (gamme $-20^{\circ} \mathrm{C}+60^{\circ} \mathrm{C}$ ). Les capteurs à ultra-sons (US) mesurant le tirant d'air sont très sensibles à la stratification de la température de l'air. La mesure de température de référence étant faite en un point, il peut résulter des écarts relativement importants en fonction de la configuration du point de mesure. Les murs verticaux en béton sont de ce point de vue mal adaptés pour la fixation du capteur. En général, les capteurs US sont moins utilisés en hydrométrie, à l'exception de certains services de prévision des crues. EDF DTG ne les utilise quasiment pas. La classe de précision du capteur bulle à bulle a été évaluée à $0,3(0,3 \%$ de l'étendue de mesure), ce qui pour un capteur dont la gamme de mesure vaut 10 mètres équivaut à $3 \mathrm{~cm}$. Pour les capteurs piezorésistifs, qui nécessitent une correction moindre consécutive à la température, la classe de précision vaut 0,1 . Les radars ont fait leur apparition sur le marché de l'hydrométrie. Ils présentent des avantages certains, comme leur non intrusion dans l'écoulement et leur insensibilité à la température. Leur classe de précision est évaluée à 0,1 . La nécessité de les éloigner de la berge (effets de bord) et la tache de réception de l'onde conditionnée par le guide d'onde peuvent pénaliser la représentativité de leur mesure par rapport à l'échelle de référence. Le nilomètre constitue la dernière innovation en matière de limnimétrie. Il utilise la conductivité de l'eau comme principe de mesure par empilage de capteurs élémentaires qui détectent leur immersion ou non. La précision est de l'ordre du $\mathrm{mm}$. Le principe est donc prometteur, mais il reste cher et mal adapté pour les hauteurs supérieures à 5 mètres.

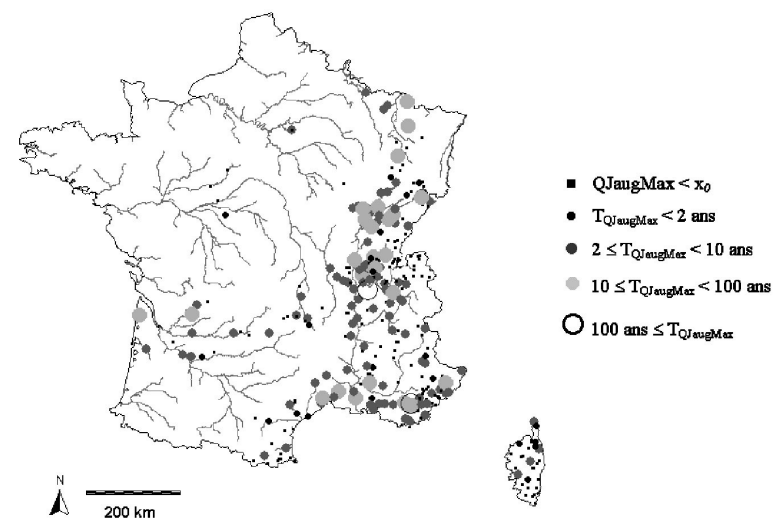

Figure 1 : Cartographie de la classe du débit maximal jaugé.

De manière pratique, pour apprécier l'incertitude résultante sur la valeur de débit, on a déterminé pour un échantillon de 87 stations d'hydrométrie gérées par EDF DTG Grenoble les hauteurs correspondant à des débits de l'ordre de la crue décennale. On montre que la moyenne s'établit à 4,1 mètres avec un maximum de 9 mètres et un minimum de 1,5 mètres. Dans ces conditions et en considérant que la majorité du parc de capteurs utilisés par EDF DTG est constituée par des capteurs bulle à bulle, on peut estimer que l'incertitude sur 
la détermination des débits de crue consécutive à l'incertitude intrinsèque du capteur de niveau, est comprise entre 0,5 et $3 \%$ avec une moyenne de $1 \%$.

\section{III.2 Les aléas de mesure}

\section{III.2.1 Variabilité de la mesure de niveau}

Si pour les rivières de plaine à crues lentes et dont le bras vif et le lit mineur ne sont pas encombrés d'obstacle l'augmentation du niveau reste régulière, il n'en va pas de même pour les rivières de montagne à crues rapides où la pente et la présence de blocs rocheux entraînent des perturbations de l'écoulement qui se traduisent par une grande variabilité du niveau. La figure 2 illustre une crue exceptionnelle enregistrée sur la Thines (affluent du Chassezac) où lors de la pointe de crue, les variations de niveau ont atteint sensiblement plus ou moins $30 \mathrm{~cm}$ pour une hauteur de l'ordre de 6 mètres $( \pm 5 \%)$. Traduites en débits, ces variations conduisent à une fourchette comprise entre 180 à $220 \mathrm{~m}^{3} / \mathrm{s}$ pour l'évaluation de la pointe de crue $( \pm 10 \%)$.

L'exemple développé ci-dessus a été choisi parmi d'autres épisodes où le signal de niveau présentait une forte variabilité. Il n'est en effet pas rare d'observer des variations de plus ou moins $5 \%$ du niveau conduisant à une incertitude de l'ordre de plus ou moins $10 \%$ sur le débit. Un simple lissage du signal suffit en général à éliminer ce type d'incertitude, à condition toutefois de disposer d'un signal suffisamment échantillonné.

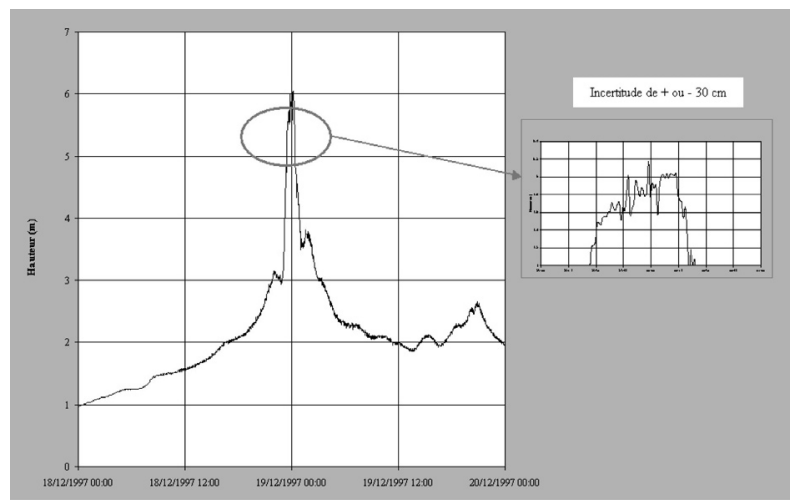

Figure 2 : Variabilité des mesures de niveau sur la Thines à Pont du Gournier (liminigramme de la crue du 19/12/1997).

\section{III.2.2 Le taux de matières en suspension}

Lorsque le principe du capteur de niveau utilisé repose sur une mesure de pression (piézorésistif ou bulle à bulle), le taux de matières en suspension contenu dans l'eau de rivière induit une incertitude supplémentaire. En effet, la densité de l'eau supposée égale à 1 est augmentée si le taux de matières en suspension est significatif. Des observations faites lors de crues sur quelques rivières des Alpes ont permis de montrer que celui-ci pouvait atteindre régulièrement $10 \mathrm{~g} / \mathrm{l}$ et monter dans certains cas jusqu'à plus de $50 \mathrm{~g} / \mathrm{l}$. On passe sous silence le cas des laves torrentielles et des coulées de boue qui sortent du champ du présent exposé. En supposant une densité égale à 2,6 pour les matières en suspension dans l'eau, la densité de l'écoulement peut atteindre et dépasser 1,03. Dans ces conditions l'erreur commise sur la mesure de niveau atteint 3\%.

On retiendra que le taux de matières en suspension de l'eau de rivière peut induire un biais dans la mesure de niveau. Celui-ci peut atteindre, voire dépasser $3 \%$. La tendance est systématiquement à la majoration. En cas de doute, on pourra effectuer un prélèvement d'échantillons et procéder au pesage des matières sèches. Cette opération est particulièrement recommandée lors des jaugeages par dilution.

\section{III.2.3 Le temps de réponse du capteur}

Les capteurs de niveau utilisés en hydrométrie doivent être capables de décrire correctement les phases de montée et de descente des crues. Les performances des capteurs, numériques ou analogiques, annoncées par les constructeurs, répondent en principe à cette exigence. Cependant, les capteurs bulle à bulle intègrent quant à eux des composants mécaniques, un compresseur, un filtre, une réserve d'air et une longueur de la ligne pneumatique, dont la défaillance ou le défaut d'entretien peuvent réduire la capacité à mesurer un gradient important. L'exemple proposé figure 2, pour la station de la Thines à Pont de Gournier équipée d'un capteur bulle à bulle, montre un gradient de montée spectaculaire : 3,07 mètres en une heure soit $5 \mathrm{~cm}$ par minute avec des pointes de $25 \mathrm{~cm}$ par minute. L'étude des restes de crue a confirmé le niveau de pointe mesuré par le capteur et rien ne laisse suspecter la moindre défaillance de ce dernier.

La figure 3 présente l'enregistrement de la crue de la Loire du 20 octobre 2001 pour la station limnimétrique de Rieutord qui contrôle $62 \mathrm{~km}^{2}$. Elle est équipée d'un capteur bulle à bulle installé dans une armoire située à une centaine de mètres de la rivière. Un examen rapide du limnigramme pourrait conduire à conclure au bon fonctionnement du matériel de mesure. En seconde lecture pourtant, la forme apparait comme suspecte dans la mesure où le gradient de descente du niveau est plus rapide que le gradient de montée. Ce constat a conduit les équipes de DTG à réaliser une reconnaissance sur le terrain dans les jours qui ont suivi la crue. Les relevés effectués à cette occasion ont montré que le niveau réellement atteint au droit de la station n'était pas égal à 3,65 mètres comme l'enregistrement l'avait tracé, mais 6,40 mètres ! Grâce à ce relevé et à une étude hydrologique, un limnigramme reconstitué peut être proposé. Le gradient de niveau reconstitué est ainsi évalué à 3,4 mètres par heure, valeur comparable à celle observée sur la Thines.

Sur cet exemple, le diagnostic du matériel a montré que la longueur du circuit pneumatique était la cause de ce dysfonctionnement. Il conduit à recommander la plus grande rigueur dans le choix des composants et l'entretien des circuits pneumatiques ainsi que le recours à l'installation d'un compresseur extérieur pour les circuits de grande longueur. A noter qu'un puits envasé produit le même type de défaut.

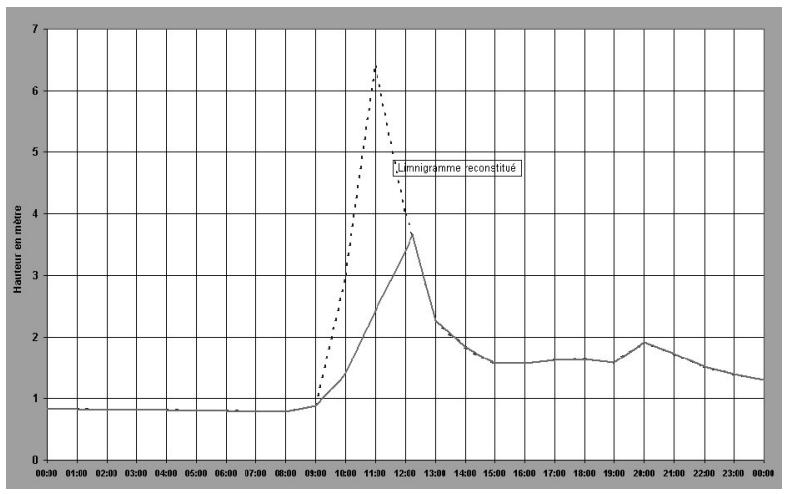

Figure 3 : Temps de réponse du capteur de niveau sur la Loire à Rieutord (crue du 20/10/2001). 


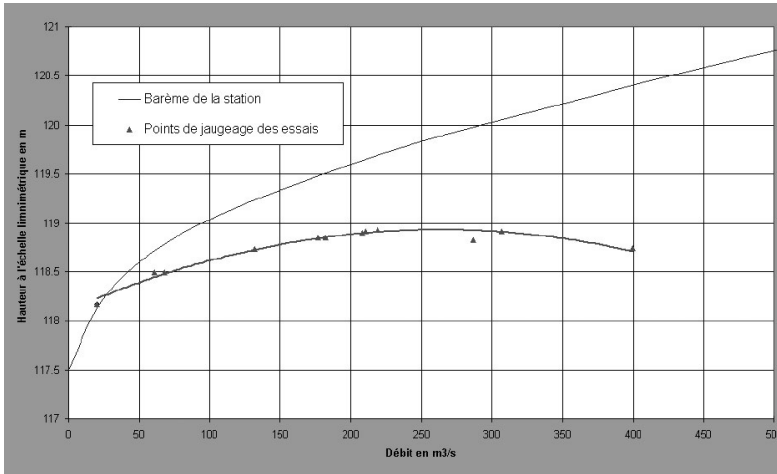

Figure 4 : Représentativité de la mesure du niveau sur l'Isère à Beaumont Monteux.

\section{III.2.4 La représentativité du niveau}

L'univocité de la relation entre la hauteur et le débit est une condition indispensable à la caractérisation d'un hydrogramme de crue à partir d'une simple mesure de hauteur. Souvent fait lors d'une reconnaissance effectuée à bas débit, le choix de l'emplacement de la section de contrôle est de ce point de vue primordial. Il peut s'avérer parfois qu'un site bien adapté pour une gamme de débits se révèle inefficace pour des débits plus élevés. La figure 4 en donne une illustration pour l'une des stations limnimétriques de Beaumont Monteux sur l'Isère dans le tronçon court-circuité du barrage. Des blocs présents dans le lit mineur, sans effet sur la ligne d'eau lorsque le débit est faible, peuvent devenir des obstacles, induire des passages en régime critique et par conséquent une baisse du niveau alors que le débit augmente. Pour éviter ce type d'aléas qui peut conduire à des erreurs importantes pour la détermination des débits de crues, on recommande d'effectuer un levé topographique (profils en long et en travers) du lit dès la reconnaissance, et la construction d'un modèle hydraulique, même sommaire.

\section{INCERTITUDES SUR LA MESURE DES DÉBITS}

Les jaugeages restent le meilleur moyen de construire une courbe de tarage. Les incertitudes associées aux différentes techniques sont décrites par la normalisation.

\section{IV.1 Exploration du champ des vitesses}

Pour l'exploration du champ des vitesses à l'aide des moulinets d'hydrométrie, la norme ISO 748 [1997] décrit la méthodologie de mesurage et propose un calcul d'incertitude. Celui-ci présente l'inconvénient majeur de donner un poids prépondérant aux nombres de verticales effectuées pour l'exploration et conduit en pratique à proposer un résultat avec une incertitude toujours supérieure à $4 \%$. Audinet [1995] considère quant à lui que l'erreur finale sur « un jaugeage bien mené dépasse rarement $5 \%$ et est presque toujours inférieure à $3 \% »$. Forray et al. [1998] proposent un diagramme de courbes enveloppes qui définit le nombre optimal de verticales en fonction de la largeur du lit et la nature de l'écoulement. Outre l'étalonnage du moulinet en fonction du type de support, du compteur d'im- pulsions et de sa base de temps, il convient aussi d'effectuer le contrôle périodique des dispositifs de mesurage des paramètres dimensionnels de la section de jaugeage lorsque la station hydrométrique est équipée d'une traille de mesure. On évite ainsi des erreurs systématiques importantes. La même précaution est à prendre pour les treuils des camions jaugeurs, des bateaux ou des cyclo-potences. Sur le plan pratique, il a été observé que la détermination de la profondeur des verticales était parfois difficile avec le saumon lorsque le tirant d'eau était très important (supérieur à 5 ou 6 mètres). Dans ce cas, le risque de maximisation de la section et donc du débit est réel. On signalera aussi les difficultés liées aux jaugeages effectués à partir de ponts composés de plusieurs portées. L'accélération du courant au voisinage des piles et la nécessité de découper le jaugeage en autant de portées que le pont en comporte rendent difficile l'exploration du champ des vitesses et introduisent une source d'incertitude supplémentaire.

L'introduction de l'ADCP (mesureur à effet Doppler) dans la panoplie du jaugeur a tendance à modifier les pratiques. L'équipe DTG de Grenoble a commencé à l'expérimenter depuis que l'évolution de la technologie autorise l'emploi de ce matériel dans des conditions de profondeurs moins contraignantes qu'au moment de son apparition au début des années 90. Les mesures concomitantes effectuées avec le traditionnel saumon ont toujours montré des résultats comparables, inférieurs à $5 \%$. Le groupe de travail constitué de différents spécialistes représentant l'hydrométrie en France devrait produire des résultats très attendus sur les performances du nouveau matériel, les incertitudes associées et la méthodologie de mise en oeuvre.

En conclusion sur ce point, on peut dire que les méthodes d'exploration du champ des vitesses restent les plus robustes lorsqu'elles peuvent être mises en œuvre, car les opérateurs peuvent contrôler de manière simple la quasi totalité des paramètres de la mesure. Dans ces conditions, l'incertitude de $5 \%$ couramment affichée pour ce type de mesures, reste un objectif souvent atteignable, y compris en crue. Certaines conditions d'écoulement plus difficiles comme la proximité des piles de pont, les vagues et les tourbillons, constituent des sources d'erreurs qui peuvent augmenter l'incertitude globale et la porter à $10 \%$. La mobilité de la section, creusement ou exhaussement durant la mesure, reste cependant un facteur d'incertitude non maîtrisable.

\section{IV.2 Jaugeages par dilution}

$90 \%$ des stations d'hydrométrie gérées par EDFDTG à Grenoble, contrôlent un bassin versant inférieur à $2000 \mathrm{~km}^{2}$. Ce constat permet de comprendre pourquoi cet organisme a développé puis préservé la compétence de ses équipes pour effectuer des jaugeages par dilution. En effet, le caractère impétueux des rivières de montagne interdit le recours à l'exploration du champ des vitesses lors des crues mais aussi parfois sur toute la gamme des régimes d'écoulement. Les conditions de sécurité du personnel restent cependant un souci majeur lors des crues, car la fréquentation des bords de rivières dans ces conditions reste un exercice dangereux. Parallèlement à la qualification de la Rhodamine Wt comme traceur au cours des années 90, EDF-DTG a introduit un calcul d'incertitude issu de la norme ISO 8466-1 [1990]. Celui-ci repose exclusivement sur la dispersion des résultats obtenus et non sur l'analyse 
des différentes sources d'erreurs. On précise que c'est la méthode par injection à débit constant qui est la plus largement utilisée par EDF DTG.

En supposant que le régime permanent est établi, que le bon mélange du traceur est réalisé et que ce dernier n'est ni absorbé, ni réduit, l'expérience montre qu'au seuil de confiance de $95 \%$, l'incertitude globale sur le débit est inférieure ou égale à $3 \%$. Cette simple proposition permet de mesurer qu'au delà du soin apporté par les opérateurs dans les opérations d'injection et de laboratoire, du suivi indispensable du matériel d'injection, le résultat final est suspendu à des critères difficilement quantifiables. En pratique, la condition de bon mélange est facilement respectée et vérifiée. Il suffit d'injecter longtemps et de prélever à une distance assez longue. L'expérience est de ce point de vue un atout incomparable. Lorsqu'une nouvelle station est mise en service, les premiers jaugeages, effectués en basses ou moyennes eaux, permettent de tester le bief de travail. On développe ci-dessous les deux autres conditions à travers des exemples.

\section{IV.2.1 Régime permanent}

Lors des crues, la condition du régime permanent est plus difficile à respecter. La figure 5(a) montre toutefois que des jaugeages par dilution ont été mis en œuvre avec succès sur la Thines pendant la crue du 6 novembre 1997. Le débit maximum mesuré de $32 \mathrm{~m}^{3} / \mathrm{s}$ représente un écoulement spécifique de $2 \mathrm{~m}^{3} / \mathrm{s}_{\mathrm{km}}$, ce qui constituait déjà un motif de satisfaction pour l'équipe gestionnaire. Le jaugeage a été réalisé dans des conditions stables. Le matériel d'injection une fois installé, il a paru judicieux aux opérateurs de continuer les opérations de jaugeages. C'est d'ailleurs devenu une consigne pour les équipes de jaugeages : il est préférable de jauger plusieurs fois la même station que de tenter un maximum sur plusieurs stations. Les jaugeages suivants ont été effectués en décrue pour des gradients horaires de l'ordre de $4 \mathrm{~m}^{3} / \mathrm{s}$. Compte tenu de la relative rapidité d'exécution de chacun des jaugeages, environ $1 / 2$ heure, ces jaugeages restent tout à fait valides. Il faut cependant rappeler que les crues excessivement rapides rendent illusoires toute mise en œuvre de cette méthode. Pour l'illustrer, on peut se reporter une fois de plus à la figure 2 qui trace le limnigramme de la crue de la Thines du 19 décembre 1997, avec une variation du niveau de plus de 3 mètres observée en une heure. On notera sur la figure 5(b) que l'extrapolation de la courbe de tarage est très importante, en comparant le débit maximum jaugé $\left(32 \mathrm{~m}^{3} / \mathrm{s}\right)$ avec celui estimé pour la crue du 19 décembre 1997 (environ $200 \mathrm{~m}^{3} / \mathrm{s}$ ).

\section{IV.2.2 Réduction du traceur}

Pour détecter la réduction ou l'absorption du traceur, EDFDTG [Vignon, 1998] a mis au point une méthode appelée « cinétique ». Elle consiste à diluer une dose de traceur dans deux ballons, l'un contenant de l'eau de la rivière, l'autre de l'eau déminéralisée, et de les agiter mécaniquement durant toute la durée du jaugeage. Ce procédé est sensé reproduire les transformations chimiques du produit dans les conditions d'agitation de la rivière. Des prélèvements d'échantillons sont régulièrement effectués et leur fluorescence est ensuite mesurée en laboratoire. La comparaison des échantillons " eau déminéralisée » et " eau de rivière » permet de déterminer un facteur de correction appelé « facteur de cinétique ». La figure 6(a) montre que le jaugeage a été effectué dans des conditions stables. C'est bien la seule certitude que nous ayons sur cette mesure. La figure 6(b) montre en effet les résultats obtenus suivant que l'on retienne ou non le facteur de cinétique, avec l'intervalle de confiance à $95 \%$ associé. La valeur la plus basse est déterminée en appliquant un facteur de cinétique de 0,61 , valeur établie à partir des échantillons de cinétique traités sur place par les opérateurs. Le taux de matières en suspension élevé ( $7 \mathrm{~g} / \mathrm{l})$ tend à justifier à lui seul cette opération. En tenant compte des intervalles de confiance à $95 \%$, l'évaluation du débit varie de 350 à $770 \mathrm{~m}^{3} / \mathrm{s}$. L'application en toute rigueur de la méthodologie conduit à valider le dépouillement qui tient compte du facteur de cinétique et donc à retenir la valeur centrée de $450 \mathrm{~m}^{3} / \mathrm{s}$ comprise entre 350 et $550 \mathrm{~m}^{3} / \mathrm{s}$ au seuil de confiance de $95 \%$. On admettra cependant que la validation finale est délicate et reste suspendue à la confiance accordée à la méthodologie. L'application d'un facteur de correction élevé, même justifié par des taux de matières en suspension importants et étayé par une méthodologie bien documentée, jette parfois un doute sur le résultat des jaugeages de crue.
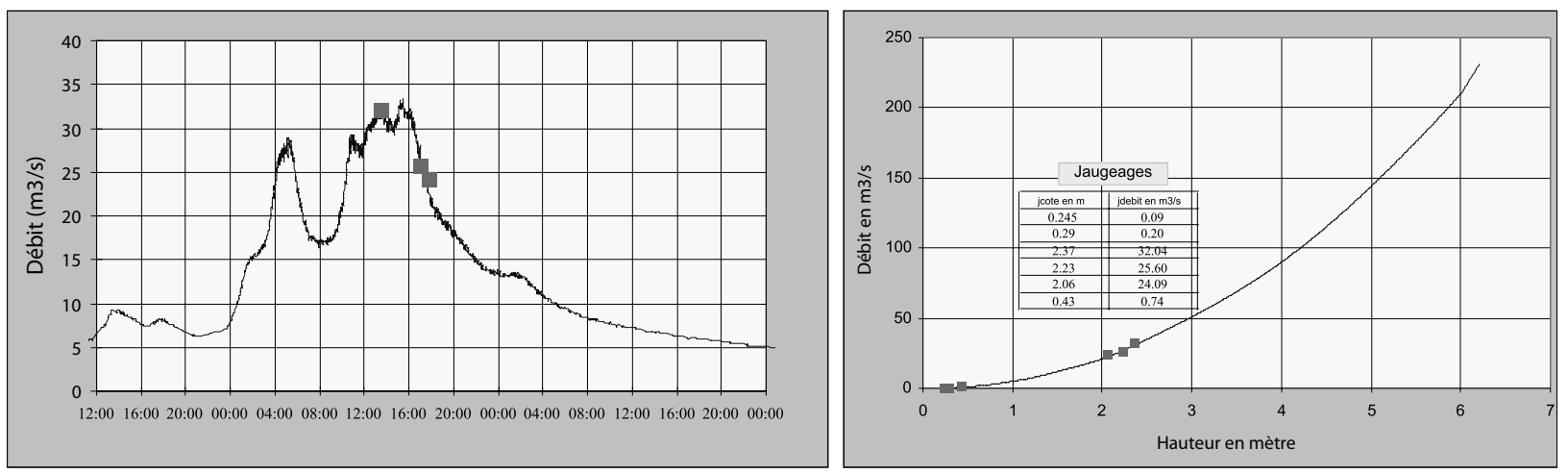

Figure 5 : Jaugeages par dilution sur la Thines à Pont du Gournier : (a) hydrogramme de la crue du 06/11/1997 ; (b) barème de tarage. 

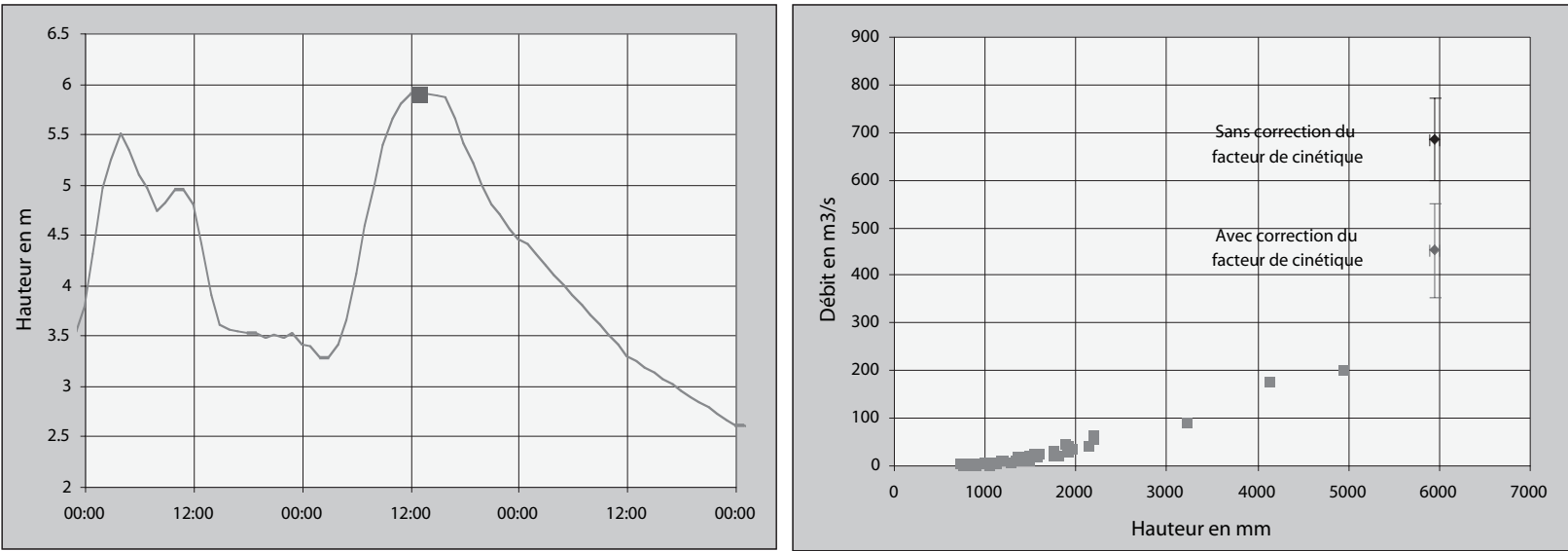

Figure 6 : Réduction du traceur sur le Buech aux Chambons

(a) limnigramme de la crue du 16/11/2001; (b) nuage 0 des jaugeages.

\section{IV.2.3 Conclusions sur la méthode par dilution}

Effectuer un jaugeage par la méthode par dilution représente souvent la seule possibilité de mesurer le débit d'une rivière. La mise en œuvre est délicate et peut s'avérer risquée pour le personnel. Elle demande du soin, sur le terrain d'abord, au laboratoire ensuite. L'absence de contrôle visuel de certains paramètres constitue la principale difficulté des opérateurs. En effet, s'il est possible et même recommandé de surveiller le débit d'injection du traceur, il n'est pas possible de vérifier directement le facteur de dilution, le bon mélange et la réduction. Seules des manipulations de laboratoire permettent d'y accéder.

La difficulté de trouver un régime permanent et la réduction du traceur restent les principales sources d'erreur. Sur la base d'un retour d'expérience effectué sur les milliers de jaugeages disponibles en archive, il paraît intéressant d'engager une étude qui consisterait à évaluer dans quelle gamme de débits les jaugeages par dilution restent fiables. Ceci permettrait d'orienter la politique de jaugeages, en concentrant les moyens sur ce qu'il est possible de faire sans introduire de doutes supplémentaires. En d'autres termes, il paraît préférable dans certains cas de bien définir la courbe de tarage en moyennes eaux par des jaugeages et de mettre quelques moyens ensuite dans la modélisation hydraulique pour mieux évaluer les crues.

\section{V q LES MOYENS DE CONTRÔLE}

Lorsqu'on n'a pas pu faire de jaugeages ou que ces derniers ne peuvent pas être validés, le recours à d'autres techniques telles que les études de cohérence hydrologiques ou les modèles hydrologiques peut s'avérer d'une grande utilité.

\section{V.1 Les ouvrages hydrauliques}

Comme le mentionne Audinet [1995], les ouvrages hydrauliques peuvent se révéler d'excellents moyens de mesure du débit. La somme algébrique des débits constitutifs du bilan de l'aménagement (débit turbiné, débit déversé, variation de réserve) permet d'accéder aux débits entrants et sortants. On peut considérer que les deux premiers termes sont en général connus avec une bonne précision, inférieure à $5 \%$. Le troisième terme, qui n'intervient justement qu'en crue, repose la plupart du temps sur des formules empiriques. Si les abaques ont été établis suivant «l'état de l'art», on peut considérer que les débits sont connus à $10 \%$ près. La figure 7 montre comment la quantification du débit de la crue du 19 décembre 1997 de la Thines, sur laquelle nous revenons une nouvelle fois, peut être confortée par une analyse faite plus en aval sur le barrage de Malarce. On dispose pour cette crue d'informations sur le débit sortant $\left(600 \mathrm{~m}^{3} / \mathrm{s}\right)$ du barrage de Ste Marguerite $\left(416 \mathrm{~km}^{2}\right)$ et sur le débit entrant $\left(1080 \mathrm{~m}^{3} / \mathrm{s}\right)$ du barrage de Malarce $\left(482 \mathrm{~km}^{2}\right)$, situés respectivement en amont et en aval de la confluence de la Thines $\left(37 \mathrm{~km}^{2}\right)$ avec le Chassezac. La différence de $480 \mathrm{~m}^{3} / \mathrm{s}$ entre ces deux débits permet d'apprécier les apports intermédiaires $\left(66 \mathrm{~km}^{2}\right)$ en terme de débit spécifique, soit $7,3 \mathrm{~m}^{3} / \mathrm{s} /$ $\mathrm{km}^{2}$. On dispose également de la valeur de la pointe de crue $\left(1371 \mathrm{~m}^{3} / \mathrm{s}\right)$ estimée par la station limnimétrique des Bertronnes $\left(507 \mathrm{~km}^{2}\right)$, ce qui permet d'apprécier les écoulements de pointe $\left(11,6 \mathrm{~m}^{3} / \mathrm{s} / \mathrm{km}^{2}\right)$ du bassin versant intermédiaire $\left(25 \mathrm{~km}^{2}\right)$ compris entre le barrage de Malarce et les Bertronnes. Cette analyse permet de conclure que l'estimation de la pointe de crue d'environ $200 \mathrm{~m}^{3} / \mathrm{s}$ sur la Thines à Pont du Gournier $\left(16,7 \mathrm{~km}^{2}\right)$ ne paraît pas irréaliste, avec un débit spécifique de $12,6 \mathrm{~m}^{3} / \mathrm{s} / \mathrm{km}^{2}$.

\section{V.2 Les modèles hydrologiques}

Comme de nombreux organismes, EDF-DTG dispose d'un modèle hydrologique «maison » appelé «MORDOR » qui, à partir des observations de pluies et de températures de l'air, permet de reconstituer un débit. La figure 8 présente en débits moyens journaliers, la crue du 13 juin 1957 sur le Guil de sinistre mémoire dont il ne reste aucune trace enregistrée. Il va de soi que ce type d'approche doit rester prudente et qu'elle ne peut constituer qu'un pis aller lorsqu'on ne dispose d'aucun élément mesuré. Elle peut également être utilisée en critique de données. 


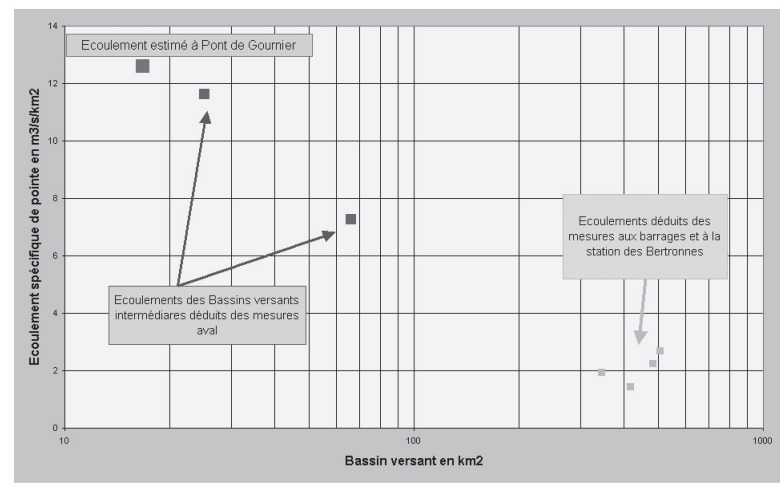

Figure 7 : Validation à l'aide d'ouvrages hydrauliques de la pointe de crue du 19/12/1997 sur le bassin de la Thine.

\section{VI — EXPERTISE HYDRAULIQUE SUR L'EXTRAPOLATION DES COURBES DE TARAGE}

\section{VI.1 Présentation des sites étudiés}

Une étude méthodologique a été réalisée par le Cemagref pour le compte du Ministère de l'Ecologie [Renouf et al., 2005], pour évaluer à partir de modélisations hydrauliques la pertinence de l'extrapolation des courbes de tarage de stations hydrométriques de la banque HYDRO. Une dizaine de sites test a été retenue, de façon à avoir une bonne couverture géographique répartie sur quatre des six grands bassins hydrographiques français : Artois-Picardie (AP), Loire-Bretagne (LB), Rhône-Méditerranée-Corse (RMC) et Seine-Normandie (SN). Le choix des sites a été réalisé en fonction de critères de disponibilité des informations topographiques (lit mineur et majeur de la rivière, géométrie des ouvrages hydrauliques) et hydrométriques (jaugeages, courbes de tarage, et éventuellement laisses de crue), en écartant les zones où la modélisation hydraulique risquait d'être trop complexe (écoulements à casier dans de larges plaines d'inondation). Le tableau 2 donne la longueur des tronçons modélisés, ainsi que la classe de fréquence associée au débit le plus fort jaugé.

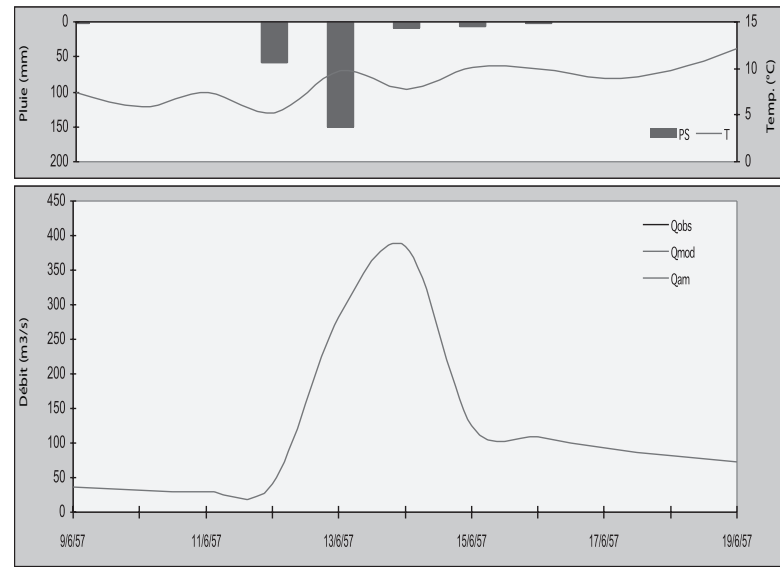

Figure 8 : Reconstitution à l'aide du modèle MORDOR de la crue du 13/06/1957 sur le bassin du Guil.

\section{VI.2 Calage des modèles hydrauliques}

La modélisation hydraulique a généralement été réalisée par un modèle 1D fluvial (MAGE), du fait de la rapidité de résolution numérique des équations de Saint Venant. Lorsque cela a été nécessaire, une modélisation plus sophistiquée a été adoptée, soit en présence de tronçons avec passage en régime torrentiel (modèle 1D torrentiel RUBARBE, station $n^{\circ} 4$ ), soit lorsque la répartition des écoulements entre le lit mineur et le lit majeur était complexe (modèle 2D RUBAR20, stations $\mathrm{n}^{\circ} 3,5,8$ et 9). L'échelle hydrométrique est le plus souvent implantée au droit d'un pont et en amont d'un seuil : il est essentiel de disposer d'une description précise de la géométrie de ces ouvrages. Le tablier des ponts n'a pas été modélisé, sauf lorsqu'il pouvait y avoir une mise en charge hydraulique de l'ouvrage, auquel cas une loi d'ouvrage (hauteur-débit) ou une modélisation géométrique par fente de Preisman (modèle 1D) a été introduite. La condition aval retenue a été la hauteur critique au droit du seuil de contrôle (lorsqu'il existe et reste en fonctionnement dénoyé) ou une condition de régime uniforme. Lorsque le tronçon modélisé n'était pas suffisamment long, un bief fictif aval a été rajouté, avec une pente moyenne égale à celle du bief et une section similaire.

Tableau 2. Sites hydrométriques étudiés

\begin{tabular}{cccccccc}
\hline $\mathbf{N}^{\mathbf{0}}$ & Rivière/Station & $\begin{array}{c}\text { Dépar- } \\
\text { tement }\end{array}$ & $\begin{array}{c}\text { Longueur } \\
\text { tronçon } \\
\mathbf{( \mathbf { k m } )}\end{array}$ & $\begin{array}{c}\text { Dist. Profils } \\
\mathbf{( m )}\end{array}$ & Bassin & $\begin{array}{c}\text { Surface } \\
\mathbf{( \mathbf { k m } ^ { 2 } )}\end{array}$ & $\begin{array}{c}\text { Période de retour } \\
\text { Jaugeage maxi }\end{array}$ \\
\hline $\mathbf{1}$ & Ain/Pont d'Ain & 01 & 10 & 500 & $\mathrm{RMC}$ & 2760 & $\mathrm{~T} \sim 5$ ans \\
\hline $\mathbf{2}$ & Ain/ Chazey-sur-Ain & 01 & 12 & 500 & $\mathrm{RMC}$ & 3630 & 2 ans $<\mathrm{T}<5$ ans \\
\hline $\mathbf{3}$ & Bouzanne/Velles & 36 & 0,4 & 20 & $\mathrm{LB}$ & 434 & $\mathrm{~T} \sim 2$ ans \\
\hline $\mathbf{4}$ & Cèze/Bessèges & 30 & 6 & 20 & $\mathrm{RMC}$ & 230 & $\mathrm{~T} \sim 5$ ans \\
\hline $\mathbf{5}$ & Gartempe/Bessines & 36 & 0,45 & 500 & $\mathrm{LB}$ & 570 & $\mathrm{~T} \sim 10$ ans \\
\hline $\mathbf{6 / 7}$ & Hérault/Gignac (Dde/Diren) & 34 & 7 & 300 & $\mathrm{RMC}$ & 1312 & 2 ans $<\mathrm{T}<5$ ans \\
\hline $\mathbf{8}$ & Hérault/Laroque & 34 & 6 & 300 & $\mathrm{RMC}$ & 760 & $\mathrm{~T} \sim 2$ ans \\
\hline $\mathbf{9}$ & Orgeval/Theil & 76 & 0,4 & 20 & $\mathrm{SN}$ & 107 & 10 ans $<\mathrm{T}<20$ ans \\
\hline $\mathbf{1 0}$ & Wimereux/Wimille & 59 & 0,5 & 50 & $\mathrm{AP}$ & 78 & $\mathrm{~T} \sim 2$ ans \\
\hline
\end{tabular}


La procédure idéale consiste à caler le modèle hydraulique en recherchant le jeu de paramètres modélisant les pertes de charge (coefficients de Strickler, lois d'ouvrages) qui minimise les écarts entre ligne d'eau simulée et observée. Une application de ce type à l'extrapolation des courbes de tarage de l'Ardèche a été réalisée par Naulet et al. [2005], pour la reconstitution des débits des plus fortes crues connues depuis 1644. La plupart du temps, on ne dispose pas de laisses de crue et le paramétrage du modèle hydraulique est obtenu d'après la configuration du terrain, à l'aide d'une table de correspondance entre la rugosité du lit et le coefficient de Manning (inverse du coefficient de Strickler) :

http://wwwrcamnl.wr.usgs.gov/sws/fieldmethods/ Indirects/nvalues/index.htm

Dans le cas présent, nous avons cherché à évaluer la pertinence du paramétrage hydraulique en exploitant les jaugeages réalisés avant et après débordement du lit mineur. Plusieurs domaines de hauteur doivent être considérés : 1/ pour les débits d'étiage, la hauteur d'écoulement devient faible par rapport au diamètre représentatif des matériaux du lit et les hypothèses de base des équations de Saint Venant ne sont plus acceptables [Smart et al., 2002] ; 2/ pour les débits mettant pleinement en eau le lit mineur, il est possible de déterminer une valeur représentative $K_{\min }$ du Strickler du lit mineur; 3 / au moment du débordement du lit mineur des pertes de charge plus importantes sont observées du fait de transferts de quantité de mouvement et de différences importantes dans le profil des vitesses [Proust, 2005 ; Proust et al., 2006] ; 4/ lorsque l'écoulement est actif dans le lit majeur, il faut introduire un coefficient $K_{m a j}$ spécifique à ce lit. Une modélisation 2D permet d'introduire plusieurs coefficients de Strickler dans le lit majeur lorsque l'occupation du sol est diversifiée, notamment dans le cas assez fréquent où les berges de la rivière sont boisées ou encombrées.

Dans l'absolu, le modèle hydraulique est d'abord calé sur le lit mineur, puis sur le lit majeur pour des hauteurs de submersions significatives $\left(H_{m a j} / H_{\min }>0,2\right)$. Dans la pratique, les plus forts jaugeages réalisés ne correspondent souvent pas à une hauteur suffisante d'écoulement dans le lit majeur pour pouvoir caler correctement le coefficient $K_{m a j}$ dans la plaine d'inondation. Aussi nous retenons sur l'Orgeval une valeur médiane de $20 \mathrm{~m}^{1 / 3} / \mathrm{s}$ et une plage de variation [15; 25], d'après la configuration du lit majeur (plaine d'herbes sans obstacles ni arbres). La figure 9(a) illustre la variation du coefficient de Strickler du lit mineur (à $K_{m a j}$ fixé) en fonction du débit pour la station de l'Orgeval à Theil : la courbe médiane correspond au calage du coefficient $K$ à partir de chaque jaugeage $\left(H_{i}, Q_{i}\right)$ et les courbes enveloppes sont relatives au calage des points $\left(H_{i}-5 \mathrm{~cm}, 1,1 Q_{i}\right)$ et $\left(H_{i}+5 \mathrm{~cm}, 0,9 Q_{i}\right)$, en considérant une erreur relative de $\pm 5 \mathrm{~cm}$ sur les hauteurs et $\pm 10 \%$ sur les débits jaugés. La droite en pointillé correspond à la limite du débordement, qui varie en fonction du Strickler utilisé pour estimer les débits. Le coefficient $K_{\min }$ est évalué à partir des quatre plus forts jaugeages avant débordement, avec une valeur médiane de $23,6 \mathrm{~m}^{1 / 3} / \mathrm{s}$ et une plage de variation $[16,9$; 30,7]. Le coefficient de Strickler diminue de façon nette au moment du débordement, avec une anomalie pour le second point, qui peut s'expliquer par une configuration particulière des berges à la date du jaugeage (travaux, encombrement). Il augmente ensuite pour les débits débordants, sans pour autant atteindre un palier, du fait de l'influence encore présente des interactions entre lit mineur et lit majeur. La figure 9(b) présente la courbe de tarage obtenue à l'issue de la modélisation hydraulique. Elle diffère de la courbe actuellement en vigueur dans l'intervalle de hauteur $[1,5 ; 2 \mathrm{~m}]$ qui correspond au débordement du lit mineur, avec des pertes de charge supplémentaires. On peut noter également l'intérêt de la modélisation hydraulique pour l'extrapolation de la courbe de tarage, avec un résultat sensiblement différent de celui auquel on arriverait par simple extrapolation graphique sur les jaugeages les plus forts disponibles. L'intervalle associé à l'incertitude sur la mesure des hauteurs et débits correspond ici à une erreur relative de l'ordre de $\pm 25 \%$ sur les débits.

\section{VI.3 Bilan sur l'extrapolation des courbes de tarage}

La synthèse des résultats obtenus sur le jeu test de dix stations hydrométriques a montré que la modélisation hydraulique permet de mieux prendre en compte les discontinuités éventuelles dans la forme des courbes de tarage. Celles-ci sont dues principalement aux changements de forme de section du lit mineur et du lit majeur, et à un champ de vitesse hétérogène au moment du débordement du lit mineur. La simple extrapolation "graphique» des courbes de tarage basée sur des jaugeages réalisés pour de faibles débits peut induire alors des erreurs importantes. Il faut souligner ici l'intérêt de disposer de jaugeages au-delà du débordement $\mathrm{du}$ lit mineur pour caler correctement la courbe de tarage en hautes-eaux. Dans certaines configurations (sites $n^{\circ} 3,5$ et 9 )
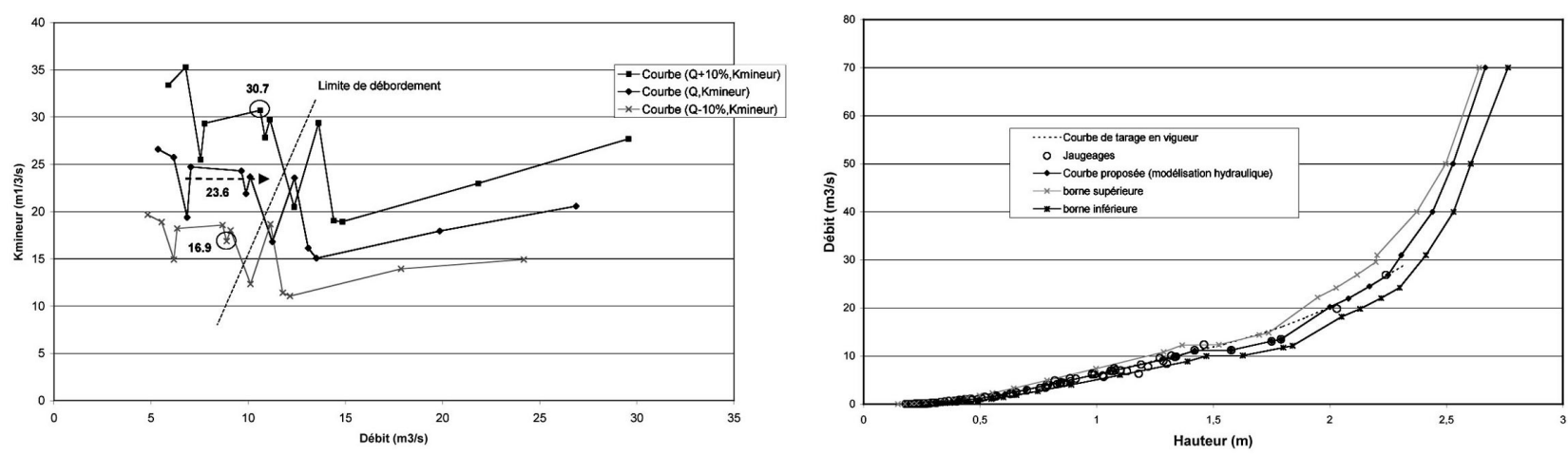

Figure 9 : Etude de la courbe de tarage de l'Orgeval à Theil (a) variation du Strickler du lit mineur en fonction du débit (b) extrapolation à l'issue de la modélisation hydraulique. 
la courbe de tarage présente un palier localisé, qui s'explique par des pertes de charge supplémentaires au moment du débordement, avec des échanges entre le lit mineur et le lit majeur. Lorsque la hauteur d'écoulement est bien supérieure à ce niveau de débordement, la courbe de tarage reprend une pente plus prononcée, les pertes de charge ayant diminué. Si aucun jaugeage supplémentaire n'est disponible, on risque d'extrapoler la courbe de tarage en prolongeant ce palier pour les fortes crues.

L'incertitude résultante sur les extrapolations issues d'un modèle hydraulique est de l'ordre de $30 \%$ pour le débit décennal des dix stations testées. Rappelons que l'hypothèse de départ a été de retenir une incertitude de $20 \%( \pm 10 \%)$ sur la mesure des débits. Cette incertitude finale peut devenir bien plus élevée lorsque la configuration hydraulique $\mathrm{du}$ site étudié est complexe (site $\mathrm{n}^{\mathrm{o}} 1$, environ $100 \%$ ) ou lorsqu'aucun jaugeage n'est disponible (site $n^{\circ} 6,60 \%$ ). Une analyse comparative sur la Gartempe à Bessines a montré que 1'exploitation des deux plus forts jaugeages (voisins de la crue décennale) a permis de réduire l'incertitude de $100 \%$ à $30 \%$. La modélisation hydraulique se révèle difficile à exploiter si les données topographiques disponibles sont insuffisantes (site $\mathrm{n}^{\circ} 10$ ) ou lorsque le fonctionnement hydraulique devient complexe en crue (mise en charge d'un pont : site $n^{\circ} 3$; ressaut hydraulique : sites $n^{\circ} 3$ et 4 ). Dans ce dernier cas, il est recommandé de choisir un modèle hydraulique adapté. On notera toutefois que ce type de configuration correspond à une ligne d'eau chahutée, ce qui induit souvent une mesure de niveau moins précise.

\section{C CONCLUSIONS ET PERSPECTIVES}

Le bilan sur 1'extrapolation des courbes de tarage en France a montré que pour la majorité des 325 stations testées, situées majoritairement sur le bassin RMC, la hauteur maximale jaugée correspond à un débit de période de retour inférieure à 2 ans. Il conviendrait de poursuivre ce travail d'analyse sur un jeu de données mieux réparti sur l'ensemble de la France, et d'en interpréter les résultats suivant le temps de réaction des bassins et de la nature des stations hydrométriques (prévision des crues, étiages, ressource ...). En l'état actuel de cette enquête, le fort niveau d'extrapolation des courbes de tarage induit une incertitude importante dans la reconstitution des débits de crue. Dans le domaine des crues jaugées, l'erreur moyenne sur la mesure des hauteurs est de l'ordre de $1 \%$ (soit $\pm 5 \mathrm{~cm}$ ). Sur les débits, elle est de 3 à $5 \%$, avec des valeurs plus importantes quand les vitesses d'écoulement ou le gradient de montée des eaux sont élevés. Dans le domaine des crues non jaugées, il est conseillé lorsque cela est possible de recouper les estimations à partir d'ouvrages hydrauliques proches ou de modèles hydrologiques. Il est recommandé de systématiquement contrôler l'extrapolation des courbes de tarage à l'aide de modélisations hydrauliques pour éviter de grossières erreurs, en particulier lorsqu'un écoulement significatif existe dans le lit majeur.

La réduction des incertitudes sur les débits de crue passe d'abord par une politique active et ciblée de jaugeages en crue, pour réduire la partie extrapolée des courbes de tarage. Elle peut ensuite être confortée par la collecte d'informations utiles au calage de modèles hydrauliques, avec notamment le relevé de laisses de crue. Enfin le retour d'expérience des services hydrométriques doit être exploité pour réduire les erreurs de métrologie et apprécier les limites inhérentes à chaque type de matériel ou chaque méthode de mesure. Le calcul complet des incertitudes sur les débits de crue nécessite d'appréhender toute la chaîne de traitement des données, depuis la mesure des hauteurs et des débits jusqu'à l'établissement de la courbe de tarage. Les réflexions menées à l'occasion de la conférence de consensus sur l'estimation du débit de la crue du Rhône à Beaucaire de décembre 2003 (http://ccbr.lyon.cemagref.fr/) ont permis d'aborder toute une série de questions méthodologiques, notamment sur les incertitudes [Cetiat, 2005], qui méritent d'être approfondies dans les prochaines années.

\section{REFERENCES}

[1] Audinet M. (1995).- - Hydrométrie appliquée aux cours d'eau. Edition Eyrolles.

[2] CETIAT, 2005. - Estimation des incertitudes: (1) de mesure de débit issus d'un jaugeage au moulinet ou à l'ADCP ; (2) des débits calculés à partir des relations hauteur-débit. Rapports d'étude $\mathrm{n}^{\circ} 2360031$ et $\mathrm{n}^{\mathrm{o}} 2460059$ pour le compte de la CNR. $80 \mathrm{p}$. et $59 \mathrm{p}$.

[3] Forray N., Auer J.C., Brochot J.F., Eraud Y., Favriau A., Ghio M., Lallement C., Lang M., Odier M., Scherer C., 1998. - Charte de qualité de l'hydrométrie; code de bonnes pratiques, Minist. Env., Groupe Qualité des Données, banque HYDRO, sept., 50 p.

[4] ISO 748, 1997. - Mesure de débit des liquides dans les canaux découverts - Méthode d'exploration du champ des vitesses.

[5] ISO 8466-1, 1990. — Qualité de l'eau - Étalonnage et évaluation des méthodes d'analyse et estimation des caractères de performance - Partie 1: Évaluation statistique de la fonction linéaire d'étalonnage.

[6] Lang M., Claudet R., 2005. - Les échelles de gravité sur les inondations : réflexion nationale et exemple dans l'Hérault. $\mathrm{La}$ Houille Blanche, $\mathrm{n}^{\circ}$ 1, pp. 52-59.

[7] Naulet R., Lang M., Ouarda T., Coeur D., Bobée B., RECKING A., Moussay D., 2005. — Flood frequency analysis on the Ardèche river using French documentary sources from the two last centuries. Journal of Hydrology, Special Issue "Applications of palaeoflood hydrology and historical data in flood risk analysis", Guest Editors G. Benito, T.B.M.J. Ouarda and A. Bárdossy, 312, pp. 58-78.

[8] Perret C., 1993. - Guide de choix des capteurs de niveaux. Document interne EDF DTG.

[9] Proust S., 2005. - Ecoulements non-uniformes en lits composés: effets de variations de largeur du lit majeur. Thèse de doctorat de 1'INSA de Lyon, $n^{\circ}$ 2005-ISAL-0083, INSA Lyon, Cemagref Lyon, $383 \mathrm{p}$.

[10] Proust, S., Rivière, N., Bousmar, D., Paquier, A., Zech, Y., and Morel, R., 2006. - Flow in compound channel with abrupt floodplain contraction. Journal of Hydraulic Engineering, à paraître.

[11] Renouf E., Lang M., Sauquet E., Paquier A., 2005. - Contrôle de la qualité des courbes de tarage de la banque HYDRO pour les débits de crue. Rapport Cemagref pour le MEDD, 53 p. + annexes (112 p.).

[12] Smart M.S., Duncan M.J., Walsh J.M., 2002. - Relatively rough flow resistance equations. J. of Hydraulic Engineering. June, 128 , pp. 568-578.

[13] Vignon P., 1998. - Mise en cuvre de la méthode cinétique lors de jaugeages par dilution à débit constant.. Document interne EDF DTG. 